\title{
INDICADORES
}

\section{MIAR: HACIA UN ENTORNO COLABORATIVO DE EDITORES, AUTORES Y EVALUADORES DE REVISTAS}

\author{
Josep-Manuel Rodríguez-Gairín, Marta Somoza-Fernández y Cristóbal Urbano
}

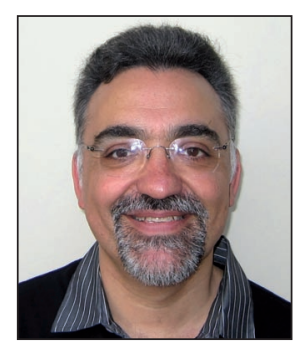

\begin{abstract}
Josep-Manuel Rodríguez-Gairín, profesor titular de la Facultat de Biblioteconomia i Documentació de la Universitat de Barcelona, es también coordinador de las aulas de informática y asesor tecnológico de la misma. Ha desarrollado la infraestructura de Temaria: Revistas digitales de biblioteconomía y documentación; BiD: Textos universitaris de biblioteconomia i documentació; MIAR: Matriz de información para la evaluación de revistas; Alehoop: Recursos en línia per elaborar treballs de recerca; EXIT: Directorio de expertos en el tratamiento de la información; IraLIS: International registry of authors-links to identify scientists. Miembro de Ciepi, ThinkEPI y del consejo técnico de E-LIS. Fundador de la empresa Kronosdoc dedicada a la asesoría y desarrollo de programas de gestión documental.
\end{abstract}

Facultat de Biblioteconomia i Documentació. Universitat de Barcelona Melcior de Palau 140, 08014 Barcelona, España rodriguez.gairin@ub.edu

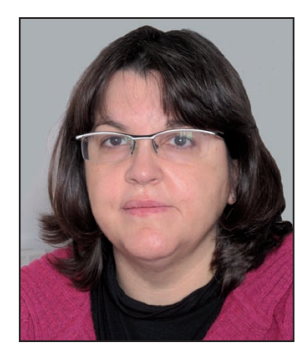

Marta Somoza-Fernández es licenciada en historia contemporánea (1989) y antropología cultural (1995) y doctora en documentación (2009) por la Universitat de Barcelona (UB). Fue responsable del Servei de Teledocumentació de l'Àrea de Ciències de la Salut de la biblioteca de la UB hasta el año 2002. Desde 1999 es profesora del Departament de Biblioteconomia i Documentació de la UB. Sus líneas de investigación se centran en bases de datos documentales, recuperación de la información, estudios bibliométricos y formación de usuarios. Es miembro del Grup d'alfabetització informacional i formació d'usuaris (AlfinCat) del Col/legi de Bibliotecaris-Documentalistes de Catalunya.

Facultat de Biblioteconomia i Documentació. Universitat de Barcelona Melcior de Palau 140, 08014 Barcelona, España msomoza@ub.edu

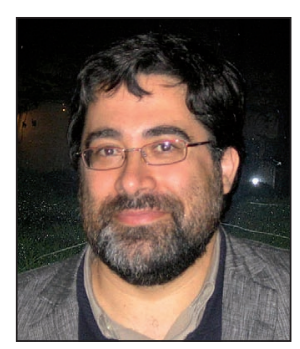

Cristóbal Urbano, profesor titular de la Facultat de Biblioteconomia i Documentació de la Universitat de Barcelona, es miembro del grupo de investigación i-Viu: información, valor i uso (2009 SGR 846) dedicado entre otras líneas a la evaluación de la investigación en ciencias humanas y sociales, y al estudio del uso de recursos bibliográficos digitales, áreas en las que ha dirigido sendas tesis doctorales. Su campo de actuación en docencia de grado son los recursos de referencia y los servicios de información. Es profesor de analítica web y de evaluación de espacios web bibliotecarios o editoriales en el Máster de Gestión de Contenidos Digitales (UB-UPF). Coordina el proyecto PCl-Aecid D/031144/10 de creación del Consorcio de Bibliotecas Universitarias de El Salvador (Cbues).

Facultat de Biblioteconomia i Documentació. Universitat de Barcelona Melcior de Palau 140, 08014 Barcelona, España urbano@ub.edu

\section{Resumen}

Se describe la evolución del proyecto MIAR (Matriu d'Informació per l'Avaluació de Revistes), un sistema originalmente diseñado para cuantificar la indización en bases de datos de revistas de humanidades y ciencias sociales. Sin embargo, a la vista del panorama de recursos de evaluación actualmente disponibles en España, los autores plantean la transformación de MIAR hacia un portal colaborativo en el que todos los interesados puedan difundir las principales características de las revistas en las que participan directa o indirectamente. Se estudia una transformación del proyecto contemplando el uso de redes sociales, sistemas de votación y de sugerencia, y la aplicación de tecnologías como open linked data que permiten una mayor difusión y socialización de los datos recogidos para cada publicación. De esta manera los datos podrían ser mejor aprovechados por los tres colectivos más directamente interesados: evaluadores, editores y autores/lectores.

\section{Palabras clave}

Revistas, Ciencias sociales, Visibilidad, Bases de datos, MIAR, Entornos colaborativos, Autogestión, Open linked data. 
Title: MIAR: A collaborative environment of editors, authors and journal reviewers

\begin{abstract}
The evolution of the MIAR project (Matriu d'Informació per l'Avaluació de Revistes) -Journal evaluation information matrix- is described. Originally MIAR was designed to quantify the indexing of humanities and social sciences journals in bibliographic data bases. Given the present availability of various journal evaluation systems in Spain, MIAR could evolve into a collaborative portal where stakeholders can share the main features of the journals in which they participate directly or indirectly. The project would use social networks, voting and commenting systems, and technologies like open linked data, thus enabling a greater dissemination and socialization of the data collected for each publication. In this way data could be better used by the three most directly concerned groups: referees, publishers and authors/readers.
\end{abstract}

\title{
Keywords
}

Journals, Social sciences, Visibility, Bibliographic databases, MIAR, Collaboration, Open linked data.

Rodríguez-Gairín, Josep-Manuel; Somoza-Fernández, Marta; Urbano, Cristóbal. "MIAR: hacia un entorno colaborativo de editores, autores y evaluadores de revistas". El profesional de la información, 2011, septiembre-octubre, v. 20, n. 5, pp. 589-595

http://dx.doi.org/10.3145/epi.2011.sep.15

\section{Introducción}

Los criterios cuantitativos para la evaluación de revistas han sido un tema controvertido ya desde hace varias décadas, tanto desde el punto de vista de los déficits de cobertura internacional de los índices de citaciones (Spinak, 1996), como de las especificidades en la evaluación de las humanidades y ciencias sociales (Borrego; Urbano, 2006) (Giménez-Toledo et al., 2007). A diferencia quizá de lo que ocurre en las ciencias experimentales, las citas como elemento de evaluación no siempre pueden constituir un único criterio determinante de calidad para la evaluación de artículos en humanidades y ciencias sociales. Por otra parte, la aplicación mecánica de clasificaciones de títulos de estas áreas, creados según una estructura de tres o cuatro niveles a manera de las clasificaciones de los cuartiles de los Journal citation reports (JCR), es motivo de polémica internacional (Pontille; Torny, 2010). Es por ello que muchos estudios consideran, junto al discutido factor de impacto de Thomson-Reuters ISI, otros elementos como la valoración de expertos (Alcaín, 2003) o índices de impacto específicos que recojan mejor la realidad española (Aleixandre et al., 2007).

Las bdds bibliográficas internacionales penalizan lo que se publica en lenguas minoritarias o lo que tiene un mercado muy acotado geográfica o culturalmente

Además, en determinadas áreas de conocimiento de las humanidades y las ciencias sociales el componente geográfico, cultural o lingüístico es parte de la identidad misma de la investigación que se hace. Sin embargo las grandes bases de datos internacionales de indización y resumen penalizan todo lo que se publica en lenguas minoritarias, o lo que tiene un "mercado" de autores / lectores muy acotado geográfica o culturalmente.
Por la gran diversidad de factores y aspectos expuestos, que configuran un panorama complicado y difícil de aprehender, es muy importante disponer de un sistema que los recoja y sistematice. Tal sistema informaría sobre el grado de difusión de las revistas en bases de datos, el cumplimiento de los criterios formales de publicación o la calidad de la revisión de artículos, que permitan tanto a lectores, potenciales autores o evaluadores establecer una valoración de la publicación más allá del grado de citas que recibe. Cada vez se hace más imperiosa la necesidad de contar con enfoques multicriterio de cobertura realmente internacional para la calificación de revistas, con vistas a la evaluación de la investigación, tal y como se ha puesto claramente de manifiesto en la reciente creación de la "Clasificación integrada de revistas científicas (CIRC)" (Torres-Salinas et al., 2010): http://epuc.cchs.csic.es/circ/que.html

http://www.elprofesionaldelainformacion.com/contenidos/2010/noviembre/circ.pdf

\section{Origen de MIAR}

En el año 2000 el Departament d'Universitats, Recerca i Societat de la Informació (Dursi) de la Generalitat de Catalunya publicó el Sistema de evaluación de la investigación en humanidades y ciencias sociales 1997-2000 (Sarhcs). Se trataba de unos listados de trece áreas temáticas que clasificaban las revistas en cuatro categorías A, B, C, D. La elaboración se había encargado desde el Cirit (Consell Interdepartamental de Recerca i Innovació Tecnològica) a comités de expertos en cada uno de los campos bajo la coordinación del Consell d'Avaluació Científico-Tècnica (Conacit, 2000).

Un grupo de investigación de la Facultat de Biblioteconomia i Documentació de la Universitat de Barcelona observó la ausencia en dichos listados de muchos de los títulos mejor posicionados en bases de datos internacionales, así como importantes incoherencias. Esta posición crítica se recogió en un artículo (Urbano, 2003) y un informe que se hizo llegar al Dursi y que dio lugar a un encargo para la revisión de dichos listados en base a la presencia de las revistas en bases de datos internacionales. 


Matriu d'Informació per a l'Avaluació de Revistes
(2011 - live)

Figura 1. Home de MIAR, http://miar.ub.es

De este encargo resultó la entrega al Dursi de una lista de 13 base de datos - una por cada una de las áreas temáticas del Sarhcs- con las que se documentó bibliométrica y bibliográficamente una amplia selección de revistas de nivel científico y académico. Realizando búsquedas bibliográficas exhaustivas tanto en el ámbito internacional como en el catalán se estableció un umbral de calidad mínimo basado en la indexación total, parcial o no indexación de las revistas en las bases de datos.

Cuando fue posible se realizó también una identificación de los títulos que tenían una temática específica coincidente con alguna de las 87 áreas universitarias de conocimiento de España; y, en caso contrario, se asignó a alguna de las 13 áreas temáticas más generales del Sarhcs, o a algún epígrafe de nueva creación. Para cada una de las revistas se calculó un indicador de difusión de los títulos en bases de datos bibliográficas (ICDS, Índice compuesto de difusión secundaria), ajustado al contexto de cada área temática.

A la vista de la ausencia de la cobertura internacional en otros proyectos similares como RESH o DICE (Giménez-Toledo et al., 2007), en los años siguientes el grupo de trabajo se planteó como objetivo la publicación en web de dichos listados con el ICDS así como su actualización continua. Para ello se contó con la ayuda del Programa de Estudios y Análisis del Ministerio de Educación y Ciencia (EA2004-0025, EA2005-0191 y EA2006-0078), y se aprovechó también para reformular su nombre dando lugar a la aparición de la sede web MIAR: Matriz de información para la evaluación de revistas.

http://miar.ub.es

En el cálculo del indicador de difusión ICDS se prima especialmente la difusión internacional de la revista en bases de datos bibliográficas especializadas, en los índices de citas del Institute for Scientific Information (Arts and Humanities Citation Index, Science Citation Index, Social Sciences Citation Index) y en Scopus, de Elsevier. Para los casos en los que no se observa presencia en bases de datos internacionales se puntúa complementariamente con otros repertorios de ámbito hispánico, como la sección selectiva "Catálogo" de
Latindex. Finalmente se completa el cálculo incorporando datos de pervivencia según el repertorio Ulrich's Periodicals Directory.

\section{Qué es MIAR}

Actualizada anualmente, la base de datos MIAR reúne información clave para la identificación y la evaluación de revistas. Éstas se agrupan en grandes áreas científicas -subdivididas a su vez en campos académicos más específicos-. El sistema crea una matriz de correspondencia entre las revistas, identificadas por su ISSN, y las bases de datos, reportorios y catálogos de bibliotecas que las indizan o incluyen. Además, se indica el vínculo a las webs de los editores e instituciones responsables de los repertorios y fuentes siempre que se dispone del mismo.

MIAR es un instrumento de apoyo para quienes han de realizar labores de evaluación: ahora disponen de datos sobre la identidad y la difusión de las revistas donde se publican los trabajos objeto de evaluación.

MIAR incluye más de 28.000 publicaciones (septiembre de 2011), para cada una de las cuales se analiza su presencia en 48 bdds y repertorios multidisciplinares y como resultado se obtiene su ICDS.

Durante los primeros años, en los que se contó con las ayudas del Ministerio de Educación de España, la actualización se realizaba con un proceso manual. Pronto se vio que el crecimiento del sistema y el futuro incierto de la financiación obligaba a plantearse un grado de automatización importante que redujera al mínimo la participación humana.

Las medidas tomadas fueron:

- Programación de agentes autónomos que periódicamente descargan la lista de revistas de cada una de las bases de datos y actualizan la tabla correspondiente en MIAR. Estos agentes pueden actuar si dicha lista contiene los ISSN y está disponible en internet en formato html, txt o pdf sin restricciones de acceso.

Por el momento estos agentes se lanzan a mano ya que los urls de las listas no son demasiado estables: suelen cambiar con cierta facilidad los nombres de los ficheros o sus ubicaciones.

- Elaboración de un formulario para dar de alta nuevas revistas que puede ser rellenado por el propio usuario que consulta MIAR. Este formulario aparece cuando la búsqueda no obtiene resultados, y requiere rellenar como campos obligatorios el ISSN y el título, así como asignar una materia académica. La revista no se introduce en el sistema hasta que es revisada por el equipo de MIAR. Si la revista está en Ulrich's, el sistema asigna automáticamente el año de inicio, el editor y el país, pero actualmente queda en manos del equipo de MIAR asignar el url y revisar o añadir los campos anteriores. 


\section{Situación actual}

En este momento podríamos decir que MIAR es un producto en busca de su audiencia y en un proceso de reflexión interna sobre su función. Los mensajes de apoyo enviados por los usuarios en los últimos años y el significativo volumen y duración de las visitas web recibidas nos confirman el interés que el recurso ha despertado en distintos colectivos y por distintas razones.

\section{Básicamente podemos hablar de:}

- Editores. Que han solicitado la inclusión de sus revistas en MIAR así como han mostrado interés en cómo hacer que aparezcan indexadas en los principales repertorios.

- Agencias de evaluación. Desde el año 2007 la Agencia de Gestió d'Ajuts Universitaris $i$ de Recerca (Agaur) de la Generalitat de Catalunya quiso que el ICDS fuera uno de los parámetros que intervienen en la elaboración del Carhust, un sistema de evaluación multicriterio de todas las revistas científicas de ciencias sociales y humanidades. De esta manera se ha establecido un acuerdo de colaboración para suministrar a la Agaur los datos del ICDS. Hay que señalar que MIAR y Carhus+ son productos diferentes e independientes. http://www10.gencat.net/agaur_ web/AppJava/castellano/a_info. jsp?contingut=carhus_2010

- Algunas universidades usan el ICDS para valorar las contribuciones en revistas que figuran en los curriculums de sus investigadores.

- Autores/investigadores. Se han recibido mensajes directos de autores e investigadores para que se incluyeran o modificaran datos de revistas en las que publican. Entendemos que en parte se debe a los procesos de evaluación a los que se están sometiendo.

- Servicios de información/documentación. En este punto mencionamos la colaboración con el servicio de bibliotecas de la Universitat Oberta de Catalunya que solicitó indicar el ICDS en los artículos de revista recogidos en su repositorio. El dato lo obtienen dinámicamente mediante una llamada rest (representational state transfer) desde el servidor del repositorio UOC al servidor de MIAR en lo que podríamos considerar el primer paso de una línea de futuro -que comentaremos más adelante- encaminada a ofrecer los datos de MIAR en xml para su reutilización en otros productos y servicios, lo que se conoce como open linked data. http://openaccess.uoc.edu/webapps/o2/help/index_es. html\#indexicds

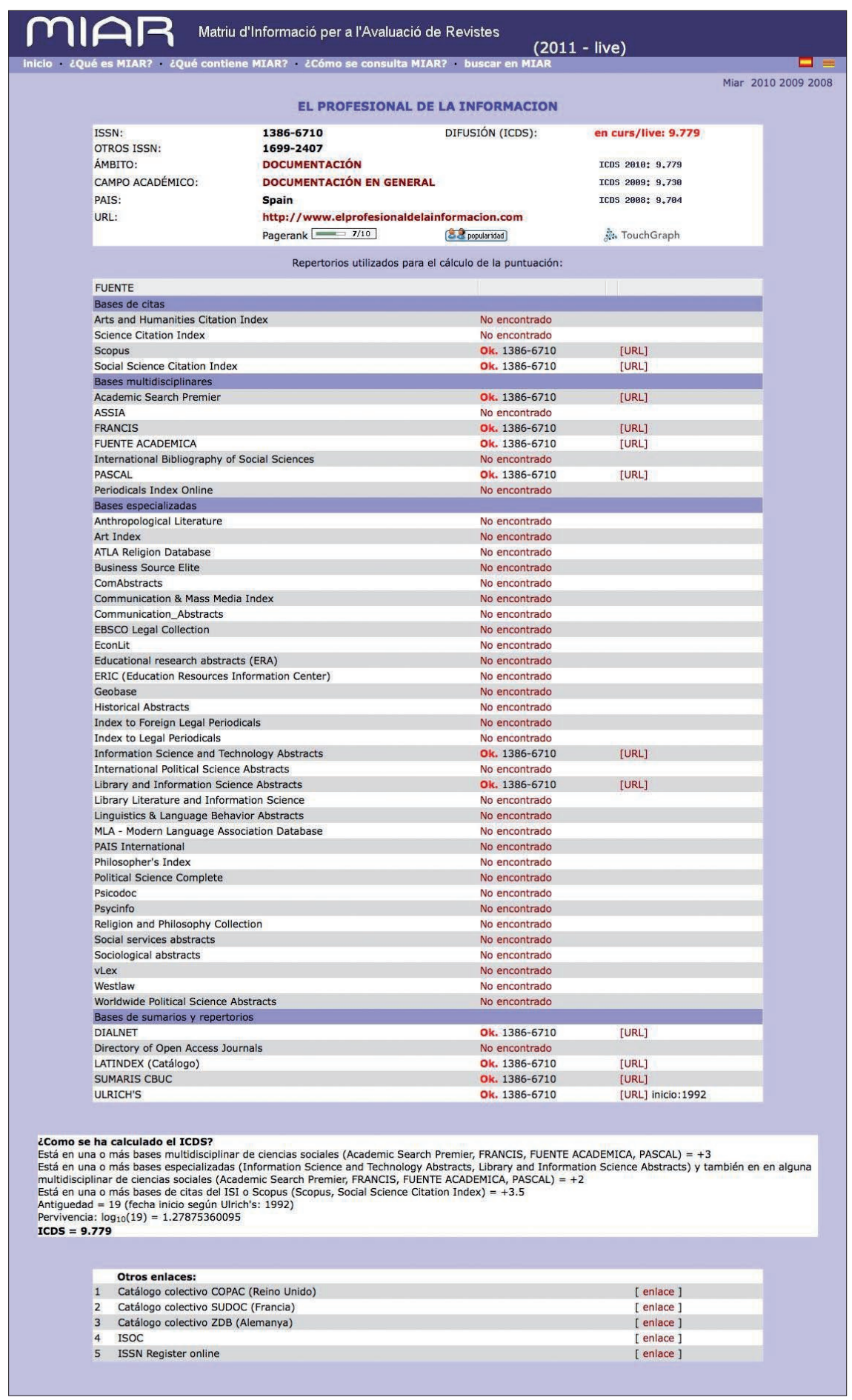

Figura 2. Ejemplo de ficha

La supervivencia de MIAR pasa por automatizar al máximo los procesos e invitar a la participación colaborativa para el mantenimiento de datos

\section{Propuestas para el futuro}

En la actualidad MIAR no cuenta con ningún tipo de subvención que permita dedicar recursos humanos a su mantenimiento, por lo que la supervivencia del mismo pasa por automatizar al máximo los procesos e invitar a la participación colaborativa en tareas de mantenimiento de datos, evidentemente sin descartar la posibilidad de obtener financiación 
de fuentes públicas o privadas para mejorar y mantener el sistema. La necesidad de una reformulación del proyecto se fundamenta en la aparición de nuevos productos con los se impondrá la cooperación o la complementariedad (Giménez-Toledo et al., 2007). A la vista del panorama de instrumentos de evaluación de revistas disponibles a día de hoy en España, se contemplan tres líneas de actuación estrechamente relacionadas entre sí para la reformulación de MIAR:

\section{Ampliación de contenidos}

En la elaboración de Carhus+ por parte de Agaur, aparte del ICDS existen otros criterios y aspectos formales como el formato, periodicidad declarada, instrucciones a los autores, metadatos, etc. Nos consta que estos datos han sido verificados manualmente accediendo a las webs de las revistas y que, en determinados casos, no han sido fáciles de localizar, han requerido una gran dedicación por parte de los evaluadores y han dado lugar a alegaciones posteriores a su publicación. A partir de esta experiencia nos planteamos que la ficha de MIAR se pueda alimentar con datos que faciliten autores o editores sobre evidencias web que acrediten dichas características formales y de proceso que se toman como indicadores de calidad de las revistas. Una propuesta inicial de campos a incluir sería:

- Periodicidad.

- Instrucciones a los autores.

- Existencia o no de palabras clave en cada artículo.

- Existencia de resumen en inglés en cada artículo.

- Sistema de revisión de los artículos.

- Tipo de comité editorial.

- Bibliografía sobre evaluación y estudios bibliométricos en los que aparece citada la revista

- Enlace permanente a la presencia de la revista en catálogos y bases de datos.

En todos los casos y dado que el sistema será autogestionado por los propios editores acreditados sin supervisión, deberá adjuntarse un enlace que sirva como prueba a fin de que el lector pueda autentificar la veracidad de la información.

\section{Entorno colaborativo y delegación}

En función de todo lo descrito anteriormente se plantea la posibilidad de transformar el sistema MIAR en un entorno colaborativo en el que todos los actores mencionados puedan enriquecer los contenidos dentro de sus posibilidades. Para ello se proponen tres niveles de gestión y delegación:

- Administrador. A este nivel tendrán acceso los responsables del proyecto y se empleará para validar los datos generales de los nuevos repertorios o bases de datos, y títulos de revista -básicamente título e ISSN- sugeridos para ser incluidos en MIAR.

- Editor. Para los editores de las publicaciones, a los que se les enviará una contraseña de modificación de datos de sus revistas -previa validación de su correo electrónicopudiendo interactuar sobre determinados campos como URL, características de la revista, etc.

- Usuario. Pensado para los usuarios finales, autores o simplemente lectores, quienes tendrán la posibilidad de sugerir nuevas revistas y bases de datos a incorporar a MIAR. Para las revistas el único requisito es que tenga un ISSN; y para las bases de datos que el listado de revistas que indiza (título e ISSN) esté libremente accesible por internet.

Igualmente se contempla la posibilidad de que puedan dejar comentarios sobre la revista (previa validación del correo electrónico - para evitar spam y garantizar una cierta identificación del responsable del comentario-).

\section{Open linked data}

Bajo el término de datos abiertos enlazados indicamos la capacidad que tienen los sistemas de generar respuestas en un formato estructurado -básicamente xml- a consultas generadas con el protocolo http en forma de enlaces. Con esta técnica los datos son recogidos por los propios servidores web e integrados en la página que va a ser mostrada al usuario final.

Aunque no es propiamente open linked data, en la actualidad MIAR devuelve una imagen con el valor del ICDS al enviar consultas. Por ejemplo, introduciendo en un navegador esta ecuación con el ISSN de la revista El profesional de la información:

http://miar.ub.es/consulta. php?issn=1386-6710\&volver= ICDS

\section{Se obtiene su ICDS: ICDS 2011: $\mathbf{9 . 7 7 9}$}

De esta forma estas imágenes con los ICDS pueden integrarse en otros servicios de terceros como en el citado repositorio institucional $\mathrm{O} 2$ de la Universitat Oberta de Catalunya (figura 3), o en la página descriptiva de las revistas del proyecto Temaria, y está a disposición de otros productos que describan revistas como podría ser Dialnet.

http://openaccess.uoc.edu/ http://www.temaria.net/revistas.php

Se está trabajando para que la totalidad de datos públicos de cada revista estén disponibles en open linked data usando rdf y los espacios de nombres de Dublin core y Prism (figura 4). Con ello se posibilita que los datos aportados por los editores, así como los propios datos de MIAR -incluido el ICDS-, puedan ser consumidos por otros servicios externos.

http://miar.ub.es/xml.php?issn=1386-6710

\section{Disponibilidad}

Está previsto hacer público el nuevo sistema con la versión MIAR 2012, en el mes de enero. Actualmente (septiembre de 2011) el sistema de edición ya está en fase de testeo para un grupo seleccionado de editores.

Los open linked data están ya activos, como puede comprobarse con los enlaces indicados en este artículo.

\section{Conclusiones}

La importancia de otros criterios complementarios a los índices de impacto medido con citas ha sido reconocida tanto por agencias de evaluación como por distintas universidades. 


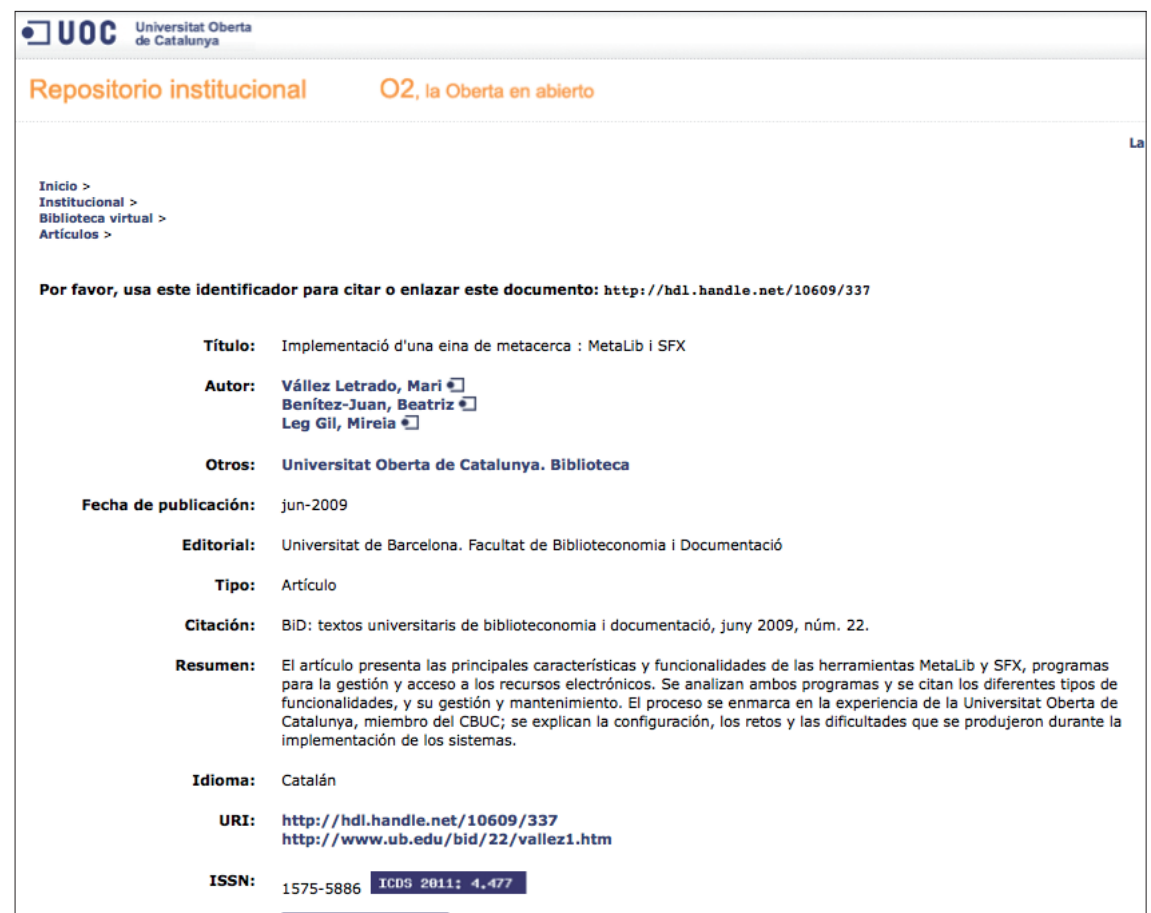

Figura 3. Ejemplo de integración: ICDS en el repositorio de la UOC

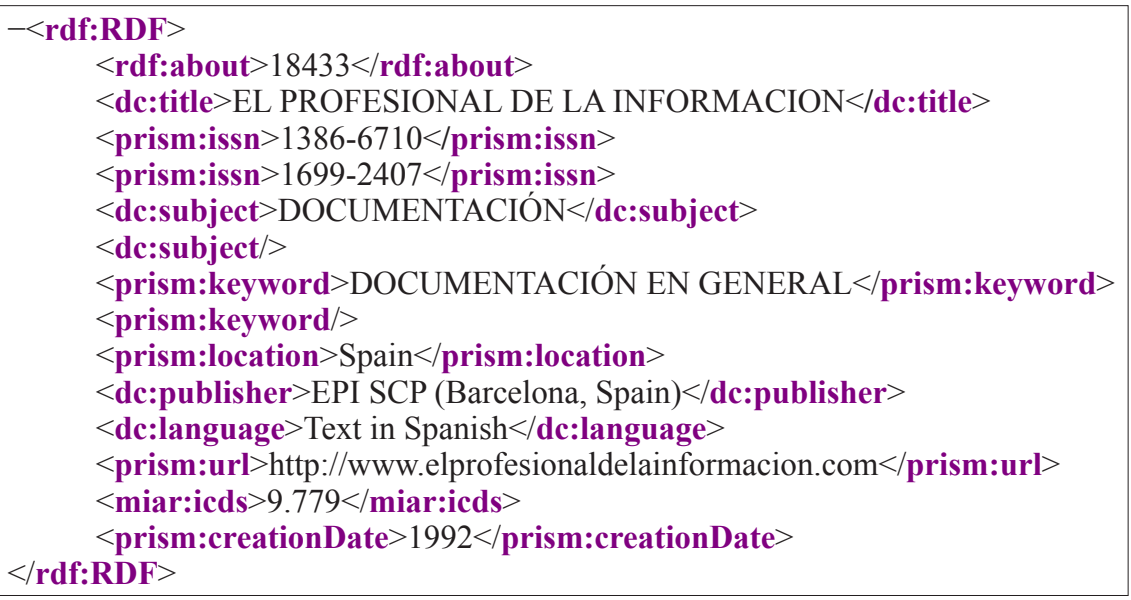

Figura 4. Ficha MIAR de una revista, etiquetada en Dublin core y Prism

Posiblemente esa sea la principal causa que ha incrementado el interés de autores y editores de revistas de humanidades y ciencias sociales para que los criterios de calidad sean fácilmente localizables y constatables, centralizados en una web accesible y usable.

Internet es sin duda el vehículo imprescindible para ofrecer este tipo de servicios, pero las tareas de mantenimiento son costosas, y difíciles de llevar a cabo si no se cuenta con financiación pública o privada. Consideramos que la propuesta de delegar el mantenimiento de los datos a editores y autores puede aliviar la carga de trabajo y dar una nueva perspectiva al servicio, aprovechando que las redes sociales y la colaboración en internet se encuentran en su máximo apogeo.

\section{Bibliografía}

Alcaín-Partearroyo, María-Dolores. Índices de impacto de las revistas españolas de ciencias sociales (biblioteconomía y documentación, psicología, psiquiatría y urbanística y orde- nación del territorio) a partir del análisis de las revistas mejor valoradas por los pares. Madrid: Consejo Superior de Investigaciones Científicas, 2003.

http://www.cindoc.csic.es/info/ inforev2.html

Aleixandre-Benavent, Rafael; Valderrama-Zurián, Juan-Carlos; GonzálezAlcaide, Gregorio. "El factor de impacto de las revistas cientificas: limitaciones e indicadores alternativos". El profesional de la información, 2007, v. 16, n. 1, pp. 4-11.

http://www.elprofesionaldelainfor macion.com/contenidos/2007/ enero/01.pdf

Bizer, Christian; Heath, Tom; BernersLee, Tim. "Linked data - The story so far". Intl journal on semantic web and information systems, 2009, v. 5, n. 3, pp. 1-22.

http://eprints.ecs.soton.ac.uk/21285/

Borrego, Ángel; Urbano, Cristóbal. "La evaluación de revistas científicas en ciencias sociales y humanidades". Información, cultura y sociedad, 2006, n. 14, pp. 11-27.

http://www.scielo.org.ar/pdf/ics/n14/ n14a02.pdf

Conacit. Proposta d'un sistema d'avaluació de la recerca a humanitats $i$ ciències socials. [Barcelona: Cirit: Departament d'Universitats Recerca i Societat de la Informació], 17 de abril de 2000.

http://web.archive.org/web/ 20010724193159/http://dursi.gencat. es/ca/re/cirit_aval.htm

Giménez-Toledo, Elea; Román-Román, Adelaida; Alcaín-Partearroyo, María-Dolores. "From experimentation to coordination in the evaluation of Spanish scientific journals in the humanities and social sciences. Research evaluation, 2007, v. 16, n. 2, pp. 137-148.

Lamarca-Langa, Genaro; Arquero-Avilés, Rosario; EstebanNavarro, Miguel-Ángel. "Evaluación de la calidad de las revistas científicas españolas en humanidades y ciencias sociales". Boletín de la Anabad. 2005, v. 55 , n. 1-2, pp. 377-391.

Pontille, David; Torny, Didier. "The controversial policies of journal ratings: evaluating social sciences and humanities". Research evaluation, 2010, v. 19, n. 5, pp 347-360.

Spinak, Ernesto. "Los análisis cuantitativos de la literatura científica y su validez para juzgar la producción latinoamericana". Boletín de la Oficina Sanitaria Panamericana, 1996, v. 120 , n. 2, pp. 139-146.

Torres-Salinas, Daniel; Bordons, María; Giménez-Toledo, Elea; Delgado-López-Cózar, Emilio; Jiménez-Contreras, Evaristo; Sanz-Casado, Elías. “Clasificación integrada de re- 
vistas científicas (CIRC): propuesta de categorización de las revistas en ciencias sociales y humana". El profesional de la información, 2010, v. 19, n. 6, pp. 675-683.

http://www.elprofesionaldelainformacion.com/contenidos/ 2010/noviembre/circ.pdf
Urbano, Cristóbal. "Avaluació de revistes i avaluació de la recerca en humanitats i ciències socials a Catalunya: aproximació a un problema". BiD: textos universitaris de biblioteconomia i documentació, 2003, n. 10.

http://www.ub.edu/bid/10urbano.htm

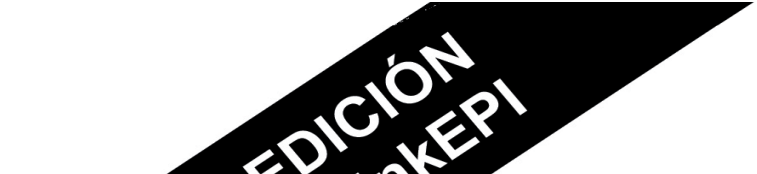 \\ 2011 \\ Análisis de tendencias en información y documentación}

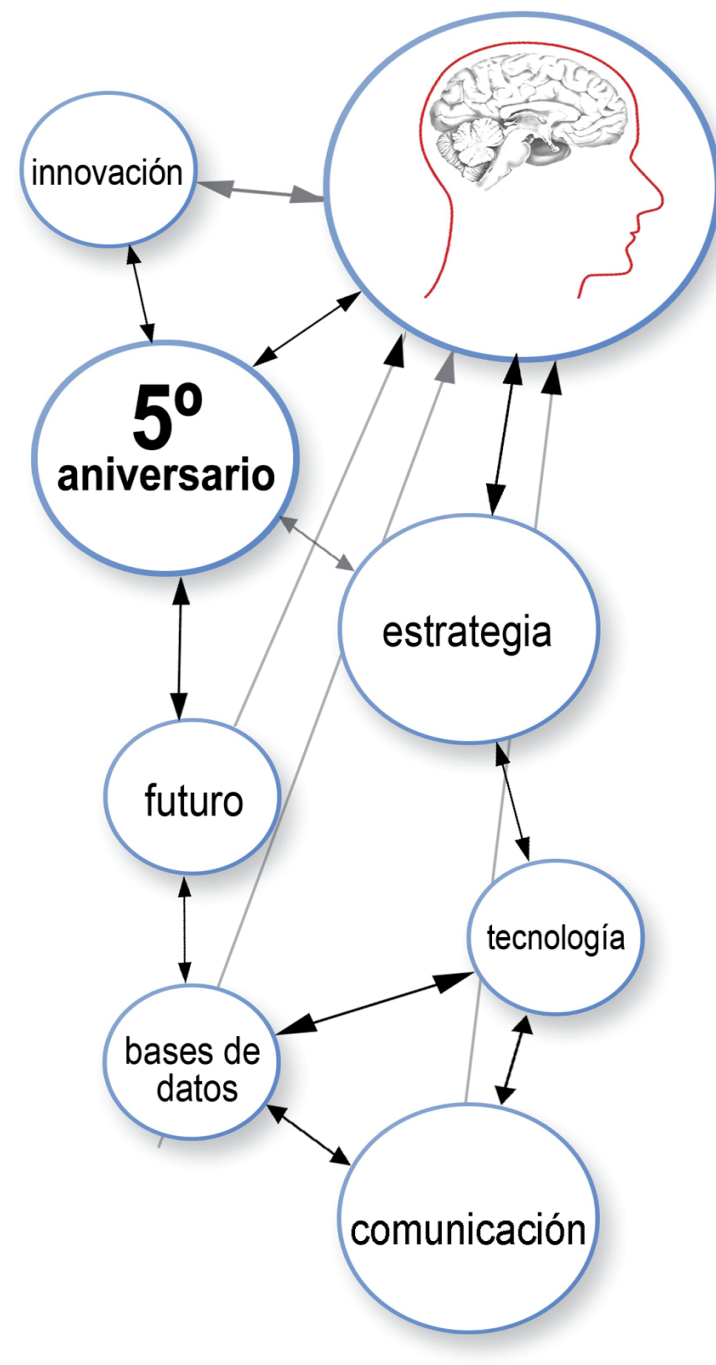

ANUARIO

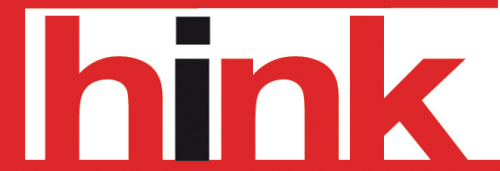

Algunos de los títulos:

Las bibliotecas, laboratorios de la administración electrónica Por Fernando Juárez-Urquijo

Proyectos de memoria ciudadana y biblioteca orientada a contenidos 2.0

Por Tomás Saorín

Bibliotecas y bibliotecarios en búsqueda de la innovación Por Roser Lozano

Ciencia 2.0: la aportación de las bibliotecas universitarias Por José-Antonio Merlo-Vega

El ecosistema de la información científica: estructura y niveles de agregación

Por Ernest Abadal y Lluís Codina

Ya puedes pasarnos tu pedido:

\section{http://www.thinkepi.net/anuario.html}

Anuario ThinkEPI 2011

Anuario ThinkEPI 2010

$$
\begin{gathered}
90,38+\text { IVA }=94 € \\
46,15+\text { IVA }=48 € \\
28,85+\text { IVA }=30 € \\
129,81+\text { IVA }=135 € \\
71,15+\text { IVA }=74 € \\
163,46+\text { IVA }=170 €
\end{gathered}
$$

Anuario ThinkEPI $2011+2010$

Anuario ThinkEPI $2010+2009$ 


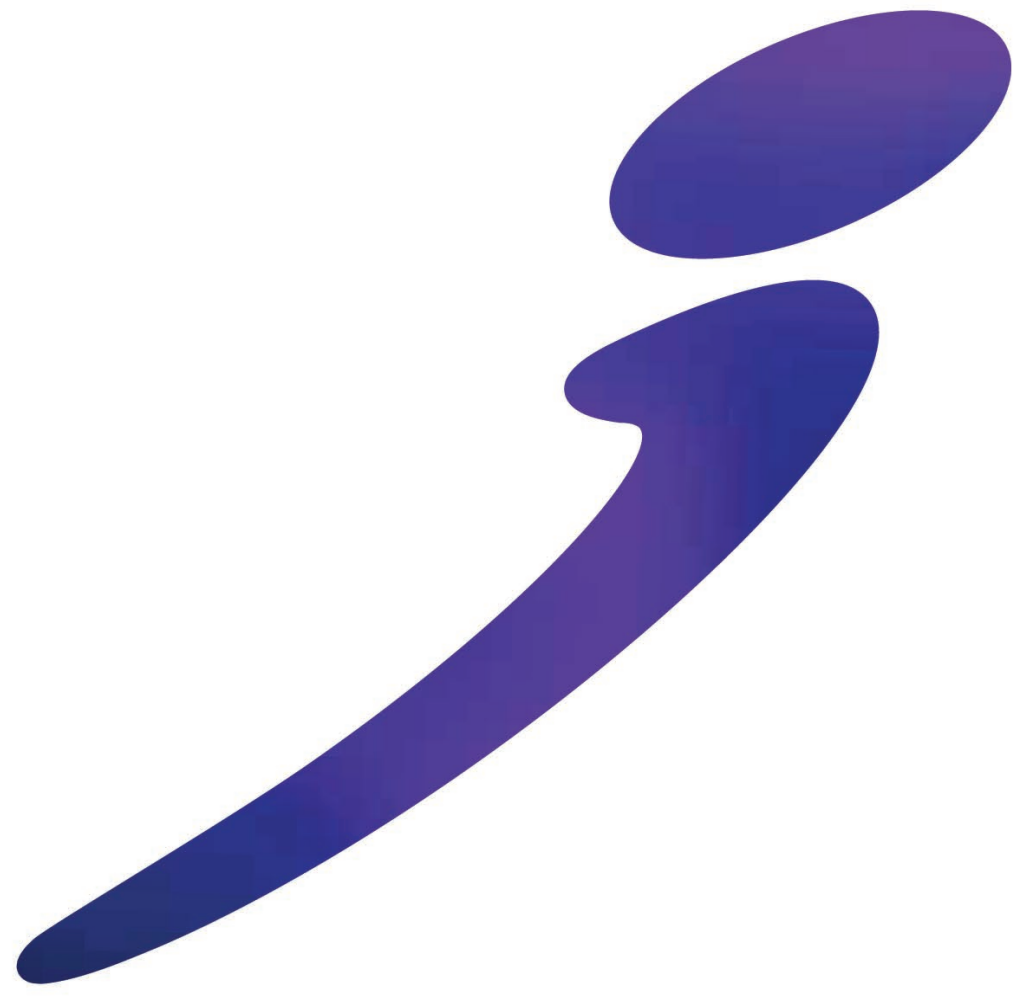

\section{ONLINE INFORMATION CONFERENCE 2011}

Information and Collaboration: Meeting the challenges of a mobile generation

This vital international forum brings together thought leaders from around the world to discuss the latest opportunities and challenges that face information professionals today.

\section{KEYNOTE SPEAKERS:}

Craig Newmark, Founder, Craigslist, USA

Rachel Botsman, Author, What's Mine is Yours: The Rise of Collaborative Consumption, Australia

\begin{tabular}{|c|c|c|c|}
\hline $\begin{array}{l}\text { Learn from our expert } \\
\text { speakers and mix and match } \\
\text { sessions to create your own } \\
\text { personalised programme. } \\
\text { Hear presentations from: } \\
\text { - British Library } \\
\text { - Chess Media Group } \\
\text { - CITI } \\
\text { - European Commission } \\
\text { - Guardian News \& Media } \\
\text { - IBM } \\
\text { - Pandia.com }\end{array}$ & $\begin{array}{l}\text { - Scottish Government } \\
\text { - The Press Association } \\
\text { - University of Sheffield } \\
\text { - Wireless Wanders } \\
\text { - Westminster Abbey } \\
\text { and any more! } \\
\text { Join your peers from over } \\
40 \text { countries to hear about: } \\
\text { The 'mobile revolution': } \\
\text { managing information for } \\
\text { people on the move }\end{array}$ & $\begin{array}{l}\text { aBooks unleashed - } \\
\text { new opportunities for } \\
\text { information professionals } \\
\text { What's new in search: } \\
\text { web, mobile, enterprise } \\
\text { and real time } \\
\text { 'Big data' - why is it } \\
\text { important and open data } \\
\text { projects delivering value }\end{array}$ & $\begin{array}{l}\text { - Skills and career } \\
\text { development to keep } \\
\text { you ahead } \\
\text { Automatic FREE } \\
\text { ENTRY to the } \\
\text { Online Information } \\
\text { Exhibition which } \\
\text { runs alongside } \\
\text { the conference }\end{array}$ \\
\hline
\end{tabular}

SUPPORTED BY

\section{a aip

